\title{
Kernos
}

Revue internationale et pluridisciplinaire de religion grecque antique

7| 1994

Varia

\section{Dennis D. HUGHES, Human Sacrifice in Ancient}

\section{Greece}

\section{Pierre Bonnechere}

Édition électronique
URL : http://journals.openedition.org/kernos/1136

DOI : 10.4000/kernos. 1136

ISSN : 2034-7871

\section{Éditeur}

Centre international d'étude de la religion grecque antique

\section{Édition imprimée}

Date de publication : 1 janvier 1994

ISSN : 0776-3824

\section{Référence électronique}

Pierre Bonnechere, « Dennis D. Hughes, Human Sacrifice in Ancient Greece », Kernos [En ligne], 7 | 1994, mis en ligne le 20 avril 2011, consulté le 24 septembre 2020. URL : http://journals.openedition.org/ kernos/1136; DOI : https://doi.org/10.4000/kernos.1136 
Dennis D. Hughes, Human Sacrifice in Ancient Greece, London-New York, Routledge, 1991. 1 vol. 14 x 22 cm, XIV + 301 p. ISBN : 0-415-03483-3.

L'A. aborde un phénomène curieux à bien des égards : il s'agit d'un sujet quelque peu choquant, presque ignoré depuis 1915, date de la parution de l'ouvrage de F. ScHWEN, Die Menschenopfer bei den Griechen und Römern, Giessen (RGVV, 15.3); mais il s'agit également d'un phénomène pour lequel nous possédons une quantité insoupçonnée de témoignages, essentiellement littéraires, qui en font une réalité de loin davantage documentée que bon nombre d'institutions antiques. Les sources figurées, également innombrables, sont ici laissées de côté.

Hughes a courageusement pris cette lourde responsabilité de traiter enfin des cas de sacrifice humain dont les archéologues ont prétendu avoir trouvé des traces certaines au cours de leurs fouilles, et les résultats de son enquête, irréprochable, sont sans appel : le sacrifice humain "archéologiquement attesté" est un concept qui doit disparaître des histoires de la religion grecque. Il ne s'agit pas de nier toute possibilité de trouver un jour une preuve archéologique d'immolation, mais d'insister sur le fait que les sources archéologiques incriminées jusqu'à présent l'ont été erronément (voir aussi Kernos, 6 [1993], p. 23$55^{1}$ ). Cette mise au point occupe au total une bonne centaine de pages et la bibliographie offre tout le loisir de constater l'énorme production qu'a assimilée l'A. en ce domaine. Ses conclusions sont particulièrement importantes dans le contexte actuel, puisque les interprétations «sacrificielles» de Sakellarakis et de Warren sont souvent présentées, aujourd'hui, comme autant de certitudes qui ont tendance à s'incruster déjà dans des ouvrages à grande diffusion (voir, par exemple,

1 À propos de la tombe 2 de la nécropole royale de Salamine, Vassos Karageorghis m'a fait parvenir quelques précisions qui méritent d'être signalées. Ainsi qu'il l'avait déjà affirmé à la suite d'une critique de P. Dikaios, en 1963, il me réaffirme que la stratigraphie du dromos était intacte, et qu'il est par conséquent impossible de considérer les inhumations faites au sommet de la couche de remblayage (in the soil of the fill within the boundaries of the dromos) comme ayant été accomplies postérieurement à la dernière inhumation dans la chambre. Il semble donc qu'il faille accepter une fois pour toutes l'idée que les squelettes fragmentaires exhumés à cet endroit aient été enterrés après la cérémonie de mise à mort des chevaux dont les vestiges furent trouvés dans la même couche, mais deux mètres plus profondément. Je répéterai donc la conclusion que j'avais tirée dans mon article : cette tombe de Salamine peut être considérée comme ayant livré un des rares cas «archéologiquement attesté» de sacrifice humain funéraire qui soit acceptable. A mon avis, cependant, l'état fragmentaire des squelettes humains demeure énigmatique dans un contexte sacrificiel, de même que la différence très nette de niveau qui séparait les restes humains et chevalins, surtout si l'on envisage une cérémonie funéraire «unique». 
M. Mastorakis et M. van Effenterre, Les Minoens. L'áge d'or de la Crète, Paris, Errance, 1991 [Coll. des Néréides], p. 75-76).

Hughes a fait la lumière sur les cas de boucs émissaires, et complété très utilement l'article de J. Bremmer, Scapegoat Rituals in Ancient Greece, in HSPh, 87 (1983), p. 299-320, tout comme son chapitre consacré aux vierges locriennes appuie l'article de F. GRAF, Die lokrischen Mädchen, in SSR, 2 (1978), p. 61-79. Sur ces deux points, la contribution de Hughes est essentielle. Le seul reproche qu'on puisse faire à l'A. est d'avoir enfermé la majorité des témoignages mythiques dans un chapitre intitulé Greek Myth, Cult and History, dans lequel il s'est limité à tenter de découvrir si les sacrifices humains avaient ou non eu lieu (selon ce qu'il avait annoncé en $\mathrm{p}$. X), sans pousser plus avant la discussion sur les origines du thème et la fréquence de son utilisation.

Bibliographie assez complète, index de sources et index général bien faits et utiles. Au total, un livre intelligent et sûr.

Pierre Bonnechere (Université de Montréal)

Marie-Chritine LECLERC, La parole chez Hésiode. À la recherche de l'harmonie perdue, Paris, Les Belles Lettres, 1993. 1 vol. $16 \times 24 \mathrm{~cm}$, 350 p. (Collection d'Études anciennes. Série grecque, 121). ISBN : 2.251.32643-X.

«Se peut-il qu'un poète chez qui le verbe poétique joue un rôle si éminent, objectivement et subjectivement, n'ait pas de la parole sinon une idée, du moins une représentation ... dans laquelle son propre verbe s'inscrirait ou de laquelle il se distinguerait ?» (p. 22). Tel est le point de départ de cette étude qui s'organise en quatre temps. Tout d'abord une étude lexicale des termes qui désignent les divers aspects de la parole, ensuite une étude stylistique de la manière dont la parole est mise en scène, en troisième lieu la recherche d'une étiologie de la parole, qui fait défaut comme telle, et enfin l'établissement de la manière dont le poète inscrit sa prestation et son savoir-faire dans la représentation de la parole ainsi mise au jour.

Hésiode ne possède pas de notion générale d'une parole abstraite, mais la perçoit dans ses manifestations : tout d'abord la parole des hommes, imparfaite, multiple, fragmentée, et la parole des dieux, efficace, oraculaire. On pressent qu'à l'origine, hommes et dieux partageaient la même parole, mais le lent processus de séparation à l'œuvre dans toute la Théogonie a affecté, déterioré le langage de l'humain, désormais contraint d'user de l'organe de la voix pour se nourrir. L'analyse de la création de Pandore, dans les Travaux, est à cet égard remarquable et montre que l'ensemble du processus se trouve 